\title{
Analysis of heavy metals in water, sediment and selected fishes of River Sutlej, Punjab, India
}

\begin{tabular}{llll}
\hline Paper received: 13.09.2017 & Revised received: 27.03.2018 & Re-revised received: 19.04.2018 & Accepted: 03.09.2018 \\
\hline
\end{tabular}

\section{Authors Info \\ S. Kaur ${ }^{1}$, P. Singh ${ }^{1 *}$, J.S. Bedi ${ }^{2}$ and S.S. Hassan ${ }^{1}$ \\ 'Department of Fisheries Resource Management, College of Fisheries, Guru Angad Dev Veterinary and Animal Sciences University, Ludhiana-141 004, India \\ ${ }^{2}$ Department of Veterinary Public Health ad Epidemiology, School of Veterinary Public Health and Zoonosis, College of Veterinary Sciences, Guru Angad Dev Veterinary and Animal Sciences University, Ludhiana-141 004, India \\ *Corresponding Author Email : prabjeet29255@yahoo.co.in}

\section{Edited by \\ Dr. Rajesh K.M.}

\section{Reviewed by}

Dr. Shiv Kumar Haragi

Dr. Aderonke Lawal

\section{Abstract}

Aim : The study aimed to assess the influence of heavy metal pollution in river Sutlej around the industrial city of Ludhiana.

Methodology : Samples of water, sediment and commercially important selected fish species were collected before (S-1) and after (S-2) the confluence of Buddah Nallah in river Sutlej for the estimation of heavy matals using Atomic Absorption Mass Spectrometer, equipped with transverse graphite furnace tube for electro thermal determination of lead, copper, chromium and zinc.

Results : The water, sediment and fish samples collected from the site, S-2 showed higher concentration of heavy metals compared site S-1. All the metals studied were found within the permissible limit of different National and International agencies, except for the lead which was found above the permissible limit in fish, Wallago attu.

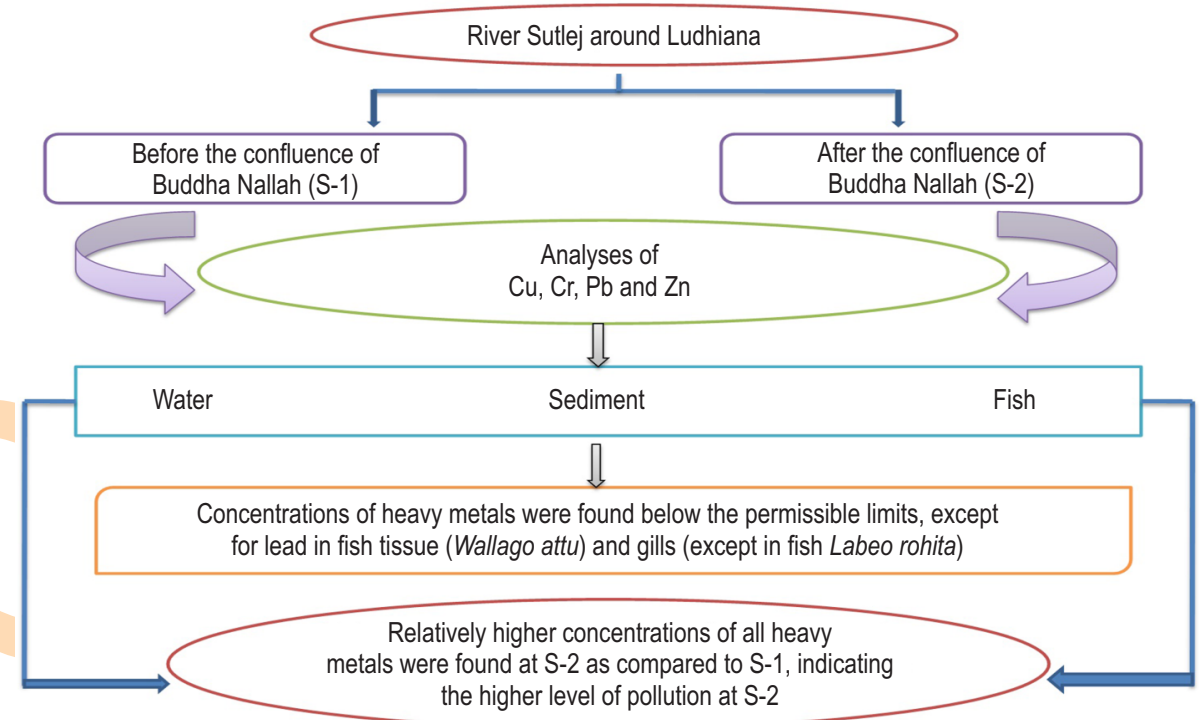

Interpretation : The results reveal that river Sutlej around Ludhiana city is under severe threat of pollution due to the discharge of industrial effluents from the Buddha Nallah.

Key words: Fish, Heavy metals, Pollution, River Sutlej

How to cite : Kaur, S., P. Singh, J.S. Bedi and S.S. Hassan: Analysis of heavy metals in water, sediment and selected fishes of river Sutlej, Punjab, India. J. Environ. Biol., 40, 322-327 (2019). 


\section{Introduction}

Contamination of water resources, sediment and biota with heavy metals is alarming due to their bioaccumulation, persistence and toxicity in industrialized nations (Al-Yousuf et al., 2000; Rashad, 2001; Ikem et al., 2003). Heavy metals, among various organic and inorganic pollutants, are widely dispersed in air, water, soil and various forms of organisms due to their nondegradable nature (Dalal et al., 2013). Human activities have significantly influenced the pollution and spatial variation of heavy metals, especially the municipal and industrial wastewater of cities and towns (Zhou et al., 2015). Heavy metals are persistent in colloidal, particulate and dissolved form in surface water. Rivers are known as the dominant pathway for the transport of metals (Miller et al., 2003). Sediments being sink for heavy metals have significant role in remobilization of contaminants in aquatic ecosystem (Zoumis et al., 2001) and are released from sediments into water and their accumulation by fish depends on several factors like solubility of heavy metals, adsorption or precipitation (lkem et al., 2003). Repid urbanization and industrialization process are big contributors to heavy metal pollution, especially in aquatic ecosystems (Singh et al., 2011). Around fifty elements are classified as heavy metals, out of these, seventeen are considered as highly toxic and relatively accessible (Singh et al., 2011). Al-Khashman (2007) and Cevik et al. (2008) reported that bio-monitoring of trace elements is essential to evaluate ecosystem.

River Sutlej has always been a large resource of capture fisheries in India. The river sustains a diverse flora and fauna, including many fish species and support livelihood of people, especially fishermen and riparian population. Aquatic biodiversity, including fish species in river Sutlej has witnessed a significant decline owing to anthropogenic activities, like fishing operations, dumping of untreated sewage, industrial and domestic waste, causing river pollution (Sharma et al., 2015). Such activities are increasing day by day and have adversely been affecting the riverine biota as well as the purity of the river water. During its course of flow through Ludhiana city, Sutlej receives direct discharge of Buddah Nallah at village Walipur Kalan. The native flora and fauna of this river is under threat due to this contamination. keeping this in view, the present study was carried out to assess the impact of heavy metal pollution in river Sutlej around the city of Ludhiana.

\section{Materials and Methods}

Sample collection: The samples of the fish (N-90), sediment $(\mathrm{N}-18)$ and water $(\mathrm{N}-18)$ collected during three seasons viz., pre-monsoon, monsoon and post-monsoon at S-1 and S-2 were transported under cooled conditions in insulated boxes to the labaroty of Guru Angad Dev Veterinary and Animal Sciences, University for further analysis. Sediment samples were collected manually from the river in plastic zip bags and stored in laboratory at room temperature till further analysis. Wallago attu (Malhi), Labeo rohita (Rohu), Channa marulius,
Aorichthys seenghala (Sanghara) and Notopterus chitala fish species were collected for heavy metal analysis in plastic zippers bags and stored at $-20^{\circ} \mathrm{C}$ till further analysis.

Digestion of water, sediment, fish tissues and fish gills: River water samples were digested manually with little modification in procedure described by Sekhon and Singh (2013). Two hundred $\mathrm{ml}$ of river water sample was taken into $250 \mathrm{ml}$ conical flask. About $10 \mathrm{ml}$ of $3 \%$ nitric acid was added to it. Acid mixed river water samples were digested manually on hot plate at $70^{\circ} \mathrm{C}$ for at least 40 minutes after which digestion was stopped. The digested sample (150 ml) was filtered through Whatman No. 1 filter paper and diluted with deionized water to make a final volume of $200 \mathrm{ml}$ in a measuring cylinder and transferred into a plastic bottle.

The river sediment was digested using a hot plate following the method of Peerzada and Rohoza (1989). The samples were oven dried at $110^{\circ} \mathrm{C}$ for $6 \mathrm{hr}$ and then grinded using a pestle mortar. The samples were homogenized and $1 \mathrm{~g}$ of the homogenized sample was sieved through 1-mm sieve. To this sample, $3 \mathrm{ml}$ of concentrated nitric acid was added and evaporated to $150^{\circ} \mathrm{C}$. The obtained residue was further subjected to drying after mixing with $5 \mathrm{ml}$ perchloric acid till a semi-dried sample was obtained. Finally, $3 \%$ hydrochloric acid (v/v) was added to the residue and sample was made $50 \mathrm{ml}$ using a volumetric flask. A blank was prepared with same procedure. Fish tissues and fish gills samples dissected out from each fish species were digested on hot plate following the procedure of FAO (1983). One gram dried and homogenized sample was weighed with digital weighing balance and $16 \mathrm{ml}$ of concentrated $\mathrm{HNO}_{3}$ and $4 \mathrm{ml}$ of $\mathrm{HClO}_{4}$ was added after pouring it into conical flask or beaker and left overnight at room temperature. The samples were then further subjected to digestion on hot plate at $120^{\circ} \mathrm{C}$ till clear solution was obtained as end point. The samples were then cooled at room temperature. Digested samples were then filtered through Whatman No. 1 filter paper and diluted with deionised water in $50 \mathrm{ml}$ measuring cylinder to make final volume up to $50 \mathrm{ml}$ and transferred to the plastic bottles for analysis.

Preparation of working solutions for AAS: Different concentrations of standards were prepared from $1 \mathrm{ppm}$ of the working solution for the calibration of atomic absorption spectrophotometer. The heavy metals ( $\mathrm{Cr}, \mathrm{Cu}, \mathrm{Pb}$ and $\mathrm{Zn}$ ) were then quantified using Graphite furnace-Atomic absorption spectrometry (GFAAS).

Statistical analyses: The results were subjected statistical analysis (One Way Analysis of Variance (ANOVA) and Duncan Multiple Range Test) to find out the significant differences in the concentration of heavy metals in fish tissue and gills. Statistical analyses was performed on SPSS-16 $(p<0.05)$ and MS-Excel.

\section{Results and Discussion}

The maximum mean concentrations of $\mathrm{Cu}(0.26$ and 0.41 $\left.\mathrm{mgl}^{-1}\right)$ and $\mathrm{Pb}\left(0.14\right.$ and $\left.0.16 \mathrm{mg} \mathrm{l}^{-1}\right)$ in water was detected during 
the pre-monsoon season whereas that of $\mathrm{Cr}\left(0.05\right.$ and $\left.0.08 \mathrm{mgl}^{-1}\right)$ and $\mathrm{Zn}\left(0.62\right.$ and $\left.0.96 \mathrm{mg} \mathrm{l}^{-1}\right)$ was detected during the postmonsoon season while minimum mean concentrations of all these elements; $\mathrm{Cu}, \mathrm{Cr}, \mathrm{Pb}$ and $\mathrm{Zn} \mathrm{(0.12} \mathrm{and} \mathrm{0.21,} 0.01$ and $0.02,0.11$ and $0.12,0.47$ and $\left.0.77 \mathrm{mg} \mathrm{l}^{-1}\right)$ were detected during the monsoon season at Site-1 and Site-2, respectively (Table 1). The mean concentrations of all these elements $(\mathrm{Cu}, \mathrm{Cr}, \mathrm{Pb}$ and $\mathrm{Zn}$ ) detected were below the permissible limits of $2 \mathrm{mg} \mathrm{l}^{-1}$, $0.1 \mathrm{mg} \mathrm{l}^{-1}, 0.05 \mathrm{mg} \mathrm{l}^{-1}$ and $5 \mathrm{mg} \mathrm{l}^{-1}$ respectively, recommended by WHO (2004), USEPA (2002) and CPCB (2000) except for lead at both the sites, which may be due to high load of industrial effluents (textile, paints and automobile) entering the river through Buddha Nallah. Higher concentrations of $\mathrm{Pb}$ detected during the present study could be due to the entry of waste from industrial activities and wastewater which is in conformity with the findings of El-Sayed et al. (2011).

Although, the concentration of $\mathrm{Pb}$ did not differ significantly, except during post-monsoon period between two sites, however its concentration was relatively higher at S-2 compared to S-1. Copper detected in higher concentration at Site-2 may be due to waste from such industrial (Automobile, Paint and Textile) holdings which tributary (Buddha Nallah) might have brought along with it. Anthropogenic sources of $\mathrm{Cr}$ emission in the surface waters are from municipal wastes, laundry chemicals, paints, leather, road runoff, agricultural runoff etc. (Dixit and Tiwari, 2008). This may hold true for the present study also as the higher concentration of $\mathrm{Cr}$ was found at Site-2 which might be due to above kind of wastes brought along with Buddah Nallah from industrial city of Ludhiana, India. The elevated levels of $\mathrm{Zn}$ in water at Site-2 may be due to the discharge of effluents from steel galvanizing industries around Ludhiana brought along with Buddah Nallah in river Sutlej. The maximum mean concentrations of $\mathrm{Cu}\left(4.23\right.$ and $18.94 \mu \mathrm{g} \mathrm{I}^{-1}$ ) and $\mathrm{Pb}$ (6.74 and $\left.7.54 \mathrm{Mg} \mathrm{I}^{-1}\right)$ in sediment was detected during the pre-monsoon season and contrarily that of $\mathrm{Cr}\left(2.78\right.$ and $\left.8.43 \mu \mathrm{gl}^{\mathrm{I}^{-1}}\right)$ and $\mathrm{Zn}$ ( 2.78 and $6.35 \mu \mathrm{g} \mathrm{I}^{-1}$ ) was detected during the post-monsoon season while minimum mean concentrations $\left(\mu \mathrm{g} \mathrm{g}^{-1}\right)$ of all these elements i.e.; $\mathrm{Cu}, \mathrm{Cr}, \mathrm{Pb}$ and $\mathrm{Zn}$ (3.89 and 11.07, 2.12 and 4.55, 4.34 and $6.98,2.70$ and $5.85 \mu \mathrm{g} \mathrm{g}^{-1}$ ) were detected during the monsoon season at S-1 and S-2, respectively (Table 2). The maximum mean concentrations of all these elements $(\mathrm{Cu}, \mathrm{Cr}, \mathrm{Pb}$ and $\mathrm{Zn}$ ) detected were below the permissible limits of $25 \mu \mathrm{g} \mathrm{g}^{-1}, 25$ $\mu \mathrm{g} \mathrm{g} \mathrm{g}^{-1}, 10 \mu \mathrm{g} \mathrm{g}^{-1}$ and $123 \mu \mathrm{g} \mathrm{g}^{-1}$ respectively, recommended by WHO (2004), USEPA (2002) and CPCB (2000) at both the sites but relatively higher concentrations were recorded at Site-2 in comparison to Site-1 which may be due to influx of industrial waste which Buddha Nallah is brings along with it.

The maximum mean concentration of $\mathrm{Cu}$ in fish tissue was observed in C. marulius (1.91 $\mathrm{\mu g} \mathrm{g}^{-1}$ wet wt.) followed by N. chitala (1.84 $\mu g g^{-1}$ wetwt.), A. seenghala(1.78 $\mu g^{-1}$ wetwt.), W. attu (1.67 $\mu \mathrm{g}^{-1}$ wet wt.) and $L$. rohita $\left(1.40 \mu \mathrm{gg}^{-1}\right.$ wet wt.) and while that of $\mathrm{Pb}$ was observed in the tissues of $W$. attu $\left(0.58 \mu \mathrm{gg}^{-1}\right.$ wet wt.), followed

Table 1: Concentration of different heavy metals in the river water $\left(\mathrm{mgl}^{-1}\right)$ at Site-1 and Site-2

\begin{tabular}{lllllll}
\hline \multirow{2}{*}{ Element } & \multicolumn{5}{c}{ Site-1 } & \multicolumn{3}{c}{ Site-2 } \\
\cline { 2 - 7 } & Pre-monsoon & Monsoon & Post-monsoon & Pre-monsoon & Monsoon & Post-monsoon \\
\hline Copper & $0.26^{\mathrm{a}, 2,3} \pm 0.01$ & $0.12^{\mathrm{c}, 6} \pm 0.001$ & $0.22^{\mathrm{b}, 4} \pm 0.001$ & $0.41^{\mathrm{a}, 1} \pm 0.001$ & $0.21^{\mathrm{c}, 4,5} \pm 0.01$ & $0.28^{\mathrm{b}, 2} \pm 0.001$ \\
Chromium & $0.02^{\mathrm{b}, 3} \pm 0.002$ & $0.01^{\mathrm{b}, 3} \pm 0.001$ & $0.05^{\mathrm{a},} \pm 0.01$ & $0.04^{\mathrm{b}, 2} \pm 0.001$ & $0.02^{\mathrm{c}, 3} \pm 0.004$ & $0.08^{\mathrm{a}, 1} \pm 0.006$ \\
Lead & $0.14^{\mathrm{a}, 1,2} \pm 0.001$ & $0.11^{\mathrm{b}, 4} \pm 0.003$ & $0.13^{\mathrm{a}, 2,3} \pm 0.002$ & $0.16^{\mathrm{a}, 1} \pm 0.01$ & $0.12^{\mathrm{b}, 3,4} \pm 0.001$ & $0.15^{\mathrm{a}, 1} \pm 0.005$ \\
Zinc & $0.53^{\mathrm{b}, 5} \pm 0.01$ & $0.47^{\mathrm{a}, 6} \pm 0.01$ & $0.62^{\mathrm{a}, 4} \pm 0.02$ & $0.89^{\mathrm{b}, 2} \pm 0.05$ & $0.77^{\mathrm{c}, 3} \pm 0.04$ & $0.96^{6^{\mathrm{a}, 1}} \pm 0.08$ \\
\hline
\end{tabular}

*Values (mean \pm standard error) in a row with different alphabetical superscripts (a, b, c) differ significantly within seasons at Site-1 and Site-2 and with different numerical superscripts $(1,2,3)$ differ significantly between sites $(P<0.05)$

Table 2 : Concentration of different heavy metals in the sediment $\left(\mu \mathrm{g} \mathrm{g}^{-1}\right)$ at S-1 and S-2

\begin{tabular}{lllllll}
\hline \multirow{2}{*}{ Element } & \multicolumn{5}{c}{ Site-1 } & \multicolumn{3}{c}{ Site-2 } \\
\cline { 2 - 7 } & Pre-monsoon & Monsoon & Post-monsoon & Pre-monsoon & Monsoon & Post-monsoon \\
\hline Copper & $4.23^{\mathrm{a}, 3} \pm 0.005$ & $3.89^{\mathrm{c}, 3,4} \pm 0.008$ & $4.08^{\mathrm{b}, 3} \pm 0.003$ & $18.94^{\mathrm{a}, 1} \pm 0.05$ & $11.07^{\mathrm{c}, 2} \pm 0.04$ & $11.54^{\mathrm{b}, 2} \pm 0.001$ \\
Chromium & $2.66^{\mathrm{b}, 4} \pm 0.008$ & $2.12^{\mathrm{c}, 4,5} \pm 0.001$ & $2.78^{\mathrm{a}, 4} \pm 0.02$ & $8.19^{\mathrm{b}, 2} \pm 0.04$ & $4.55^{\mathrm{c}, 3} \pm 0.006$ & $8.43^{\mathrm{a}, 1} \pm 0.005$ \\
Lead & $6.74^{\mathrm{a}, 2} \pm 0.003$ & $4.34^{\mathrm{c}, 4} \pm 0.008$ & $5.27^{\mathrm{b}, 3} \pm 0.006$ & $7.54^{\mathrm{a}, 1} \pm 0.009$ & $6.98^{\mathrm{c}, 2} \pm 0.008$ & $7.35^{\mathrm{b}, 1} \pm 0.002$ \\
Zinc & $2.71^{\mathrm{b}, 3} \pm 0.002$ & $2.70^{\mathrm{b}, 3} \pm 0.005$ & $2.78^{\mathrm{a}, 3} \pm 0.01$ & $6.32^{\mathrm{b}, 1} \pm 0.008$ & $5.85^{\mathrm{c}, 2} \pm 0.008$ & $6.35^{a^{\mathrm{a}, 1}} \pm 0.008$ \\
\hline
\end{tabular}

*Values (mean \pm standard error) in a row with different alphabetical superscripts (a, b, c) differ significantly within seasons at Site-1 and Site-2 and with different numerical superscripts $(1,2,3)$ differ significantly between sites $(P<0.05)$ 
Table 3: Concentration of different heavy metals in the fish tissue ( $\mu \mathrm{g} \mathrm{g}^{-1}$ wet wt.) at S-1 and S-2

\begin{tabular}{|c|c|c|c|c|c|c|c|}
\hline \multirow[t]{2}{*}{ Fish species } & \multirow[t]{2}{*}{ Element } & \multicolumn{3}{|c|}{ Site-1 } & \multicolumn{3}{|c|}{ Site-2 } \\
\hline & & Pre-monsoon & Monsoon & Post-monsoon & Pre-monsoon & Monsoon & Post-monsoon \\
\hline \multirow[t]{4}{*}{ Labeo rohita } & Copper & $1.21^{\mathrm{a}, 2} \pm 0.007$ & $0.84^{b, 4} \pm 0.008$ & $1.08^{\mathrm{ab}, 3} \pm 0.004$ & $1.40^{\mathrm{a}, 1} \pm 0.22$ & $0.88^{c, 4} \pm 0.003$ & $1.19^{b, 2} \pm 0.009$ \\
\hline & Chromium & $N D^{* *}$ & $N D^{* *}$ & $N D^{* *}$ & $N D^{* *}$ & $N D^{* *}$ & $N D^{* *}$ \\
\hline & Lead & $0.08^{\mathrm{a}, 3} \pm 0.003$ & $0.01^{c, 4} \pm 0.007$ & $0.04^{b, 3,4} \pm 0.008$ & $0.19^{a, 1} \pm 0.005$ & $0.02^{c, 4} \pm 0.003$ & $0.17^{0,2} \pm 0.008$ \\
\hline & Zinc & $0.85^{b, 3} \pm 0.005$ & $0.73^{\mathrm{c,4} .4} \pm 0.003$ & $0.90^{0,2} \pm 0.008$ & $0.93^{\mathrm{b}, 2} \pm 0.003$ & $0.91^{c, 2} \pm 0.002$ & $1.08^{\mathrm{a}, 1} \pm 0.007$ \\
\hline \multirow[t]{4}{*}{ Wallago attu } & Copper & $1.28^{\mathrm{a}, 4} \pm 0.002$ & $0.78^{\mathrm{b}, 5} \pm 0.006$ & $1.27^{\mathrm{a}, 4} \pm 0.03$ & $1.67^{a, 1} \pm 0.007$ & $1.36^{c, 3} \pm 0.003$ & $1.62^{\mathrm{b}, 2} \pm 0.007$ \\
\hline & Chromium & $N D^{* *}$ & $N D^{* *}$ & $N D^{* *}$ & $N D^{* *}$ & $N D^{* *}$ & $N D^{* *}$ \\
\hline & Lead & $0.39^{a, 3} \pm 0.001$ & $0.17^{c, 5} \pm 0.006$ & $0.26^{\mathrm{b}, 4} \pm 0.003$ & $0.58^{\mathrm{a}, 1} \pm 0.002$ & $0.39^{c, 3} \pm 0.001$ & $0.45^{\mathrm{b}, 2} \pm 0.001$ \\
\hline & Zinc & $1.03^{\mathrm{b}, 2} \pm 0.003$ & $0.85^{c, 3,4} \pm 0.008$ & $1.07^{\mathrm{a}, 2} \pm 0.008$ & $1.15^{b, 1} \pm 0.008$ & $0.95^{c, 3} \pm 0.003$ & $1.16^{a, 1} \pm 0.002$ \\
\hline Aorichthys & Copper & $1.57^{\mathrm{a}, 2} \pm 0.003$ & $1.25^{c, 4} \pm 0.006$ & $1.44^{b, 3} \pm 0.008$ & $1.78^{\mathrm{a}, 1} \pm 0.008$ & $1.40^{c, 3} \pm 0.002$ & $1.71^{\mathrm{b}, 1} \pm 0.003$ \\
\hline \multirow[t]{3}{*}{ seenghala } & Chromium & $N D^{* *}$ & $N D^{* *}$ & $N D^{* *}$ & $N D^{* *}$ & $N D^{* *}$ & $N D^{* *}$ \\
\hline & Lead & $0.32^{\mathrm{a}, 1,2} \pm 0.004$ & $0.10^{c, 4} \pm 0.005$ & $0.23^{\mathrm{b}, 3} \pm 0.005$ & $0.36^{\mathrm{a}, 1} \pm 0.005$ & $0.23^{c, 3} \pm 0.008$ & $0.34^{\mathrm{b}, 1} \pm 0.008$ \\
\hline & Zinc & $1.29^{\mathrm{b}, 2} \pm 0.003$ & $0.93^{c, 4,5} \pm 0.006$ & $1.31^{0,1} \pm 0.007$ & $1.23^{a, 3} \pm 0.008$ & $0.95^{b, 4} \pm 0.008$ & $1.24^{a, 3} \pm 0.008$ \\
\hline \multirow[t]{4}{*}{ Channa marulius } & Copper & $1.89^{a, 1} \pm 0.004$ & $1.71^{1,3} \pm 0.002$ & $1.84^{\mathrm{b}, 2} \pm 0.007$ & $1.91^{a, 1} \pm 0.003$ & $1.70^{c, 3} \pm 0.009$ & $1.82^{\mathrm{b}, 2} \pm 0.004$ \\
\hline & Chromium & $N D^{* *}$ & $N D^{* *}$ & $N D^{* *}$ & $N D^{* *}$ & $N D^{* *}$ & $N D^{* *}$ \\
\hline & Lead & $0.34^{a, 1} \pm 0.001$ & $0.19^{c, 6} \pm 0.004$ & $0.30^{b, 2} \pm 0.009$ & $0.28^{\mathrm{a}, 2,3} \pm 0.005$ & $0.21^{c, 4,5} \pm 0.005$ & $0.23^{\mathrm{b}, 2} \pm 0.008$ \\
\hline & Zinc & $1.11^{0,3} \pm 0.002$ & $0.88^{c, 5} \pm 0.002$ & $1.13^{\mathrm{a}, 3} \pm 0.005$ & $1.32^{\mathrm{b}, 2} \pm 0.007$ & $1.09^{c, 4} \pm 0.008$ & $1.44^{a, 1} \pm 0.008$ \\
\hline \multirow[t]{4}{*}{ Notopterus chitala } & Copper & $1.73^{\mathrm{a}, 3} \pm 0.001$ & $1.52^{b, 4,5} \pm 0.001$ & $1.72^{\mathrm{a}, 3} \pm 0.001$ & $1.84^{a, 1} \pm 0.004$ & $1.54^{c, 4} \pm 0.002$ & $1.79^{\mathrm{b}, 2} \pm 0.006$ \\
\hline & Chromium & $N D^{* *}$ & $N D^{* *}$ & $N D^{* *}$ & $N D^{* *}$ & $N D^{* *}$ & $N D^{\star *}$ \\
\hline & Lead & $0.26^{a, 3} \pm 0.005$ & $0.19^{c, 5} \pm 0.003$ & $0.23^{b, 3,4} \pm 0.003$ & $0.41^{a, 1} \pm 0.002$ & $0.34^{c, 2} \pm 0.001$ & $0.39^{b, 1,2} \pm 0.001$ \\
\hline & Zinc & $1.09^{b, 3} \pm 0.006$ & $0.88^{c, 4} \pm 0.007$ & $1.13^{\mathrm{a}, 1,2} \pm 0.007$ & $1.09^{b, 3} \pm 0.009$ & $0.90^{c .4} \pm 0.008$ & $1.15^{\mathrm{a}, 1} \pm 0.008$ \\
\hline
\end{tabular}

*Values (mean \pm standard error) in a row with different alphabetical superscripts ( $a, b, c)$ differ significantly within seasons at Site-1 and Site-2 and with different numerical superscripts $(1,2,3)$ differ significantly between sites $(P<0.05)$. ${ }^{*} \mathrm{ND}-$ Not detected *Statistical analysis was carried out individually for each fish species within and between the sites

by N. Chitala (0.41 $\mu g^{-1}$ wetwt.), A. seenghala ( $0.36 \mu g^{-1}$ wetwt.), C. marulius ( $0.34 \mu \mathrm{g} \mathrm{g}^{-1}$ wet wt.) and $L$. rohita $\left(0.19 \mu \mathrm{g} \mathrm{g}^{-1}\right.$ wet wt.) during pre-monsoon period whereas that of $\mathrm{Zn}$ was observed in $\mathrm{C}$. marulius (1.44 $\mathrm{\mu g} \mathrm{g}^{-1}$ wet wt.), followed by $A$. seenghala (1.31 $\mu \mathrm{gg}^{-1}$ wet wt.), W. attu (1.16 $\mu g^{-1}$ wet wt.), N. chitala (1.15 $\mu g^{-1}$ wet wt.) and $L$. rohita (1.08 $\mu \mathrm{g} \mathrm{g}^{-1}$ wet wt.) during post-monsoon season at Site-2 (Table 3). Chromium was not detected in any of the fish species (in tissue samples) during the present study which may be due to lower concentration of $\mathrm{Cr}$ in water or due to its elimination through feces (Misra etal., 2005).

The maximum mean concentrations of all these elements $(\mathrm{Cu}, \mathrm{Pb}$ and $\mathrm{Zn}$ ) were found to be below the permissible limits of $30 \mu \mathrm{g} \mathrm{g}^{-1}, 0.5 \mu \mathrm{g} \mathrm{g}^{-1}$ and $40 \mu \mathrm{g} \mathrm{g}^{-1}$ respectively, recommended by FAO/WHO (1989) except, W. attu in which the maximum mean concentration (0.58) of $\mathrm{Pb}$ was found above the permissible of 0.5 $\mu \mathrm{g} \mathrm{g}{ }^{-1}$ during pre-monsoon period at S-2. Lead is a naturally occurring substance whose environmental concentrations increase mainly due to the anthropogenic activities which include battery manufacturing, mining of base metal, $\mathrm{Pb}$-based paints and leaded gasoline (Monteiro et al., 2011).). Lead being a persistent heavy metal has been categorized as a highly hazardous material (Sfakianakis et al., 2015). The mean concentration of these elements in fish tissue differed significantly within the seasons and between the sites $(p<0.05)$ for all the fish species observed during the study period except for Notopterus chitala where no significant differences were witnessed in $\mathrm{Zn}$ concentration between S-1 and S-2 $(p<0.05)$. The trend of seasonal variations in the concentration of heavy metals in fish tissue was found in the following order $\mathrm{Pb}>\mathrm{Cu}>\mathrm{Zn}$ according to their permissible limits. Copper plays an important role in cellular metabolism as a key constituent of metabolic enzymes and an essential trace metal (Monteiro et al., 2009). Chronic toxic effects may lead to poor growth, decreased immunity, shortening of life span, problems associated with reproduction, low fertility and changes in the appearance and behavior of aquatic fauna especially fish as reported by (Yacoub and Gad, 2012).

The main detrimental changes which $\mathrm{Zn}$ may cause include mortality, retardation of growth, changes in respiration and cardiac activity, spawning inhibition and several associated effects which may pose threat to survival of fish. The elevated levels of $\mathrm{Zn}$ in water at Site-2 may be due to discharge of effluents from steel galvanizing industries around Ludhiana into the Buddah Nallah in river Sutlej. Due to the tendency of bioaccumulation the heavy metal pollution is posing a serious threat to aquatic biota (Reyahi-Khoram et al., 2016). Paulino et al. (2014) reported that exposure at chronic levels to multiple water contaminants, affects the resident biota, even at low levels, including fish. 
Table 4 : Concentration of different heavy metals in the fish gills ( $\mu \mathrm{g} \mathrm{g}^{-1}$ wet wt.) at S-1 and S-2

\begin{tabular}{|c|c|c|c|c|c|c|c|}
\hline \multirow[t]{2}{*}{ Fish species } & \multirow[t]{2}{*}{ Element } & \multicolumn{3}{|c|}{ Site-1 } & \multicolumn{3}{|c|}{ Site-2 } \\
\hline & & Pre-monsoon & Monsoon & Post-monsoon & Pre-monsoon & Monsoon & Post-monsoon \\
\hline \multirow[t]{4}{*}{ Labeo rohita } & Copper & $1.27^{\mathrm{a}, 2} \pm 0.02$ & $0.91^{b, 4} \pm 0.001$ & $1.24^{a, 2} \pm 0.006$ & $1.34^{a, 1} \pm 0.009$ & $1.18^{c, 3} \pm 0.002$ & $1.32^{b, 1} \pm 0.004$ \\
\hline & Chromium & $0.12^{\mathrm{b}, 2} \pm 0.001$ & $0.09^{c, 3} \pm 0.001$ & $0.13^{\mathrm{a}, 1} \pm 0.004$ & $0.13^{a, 1} \pm 0.006$ & $0.12^{\mathrm{b}, 2} \pm 0.004$ & $0.14^{a, 1} \pm 0.006$ \\
\hline & Lead & $0.19^{a, 2,3} \pm 0.002$ & $0.02^{c, 6} \pm 0.001$ & $0.17^{0,2} \pm 0.006$ & $0.37^{a, 1} \pm 0.009$ & $0.06^{c, 5} \pm 0.006$ & $0.22^{\mathrm{b}, 2} \pm 0.004$ \\
\hline & Zinc & $1.22^{\mathrm{b}, 4} \pm 0.006$ & $1.16^{c, 5,6} \pm 0.003$ & $1.30^{a, 3} \pm 0.008$ & $1.55^{\mathrm{b}, 2} \pm 0.005$ & $1.19^{c, 5} \pm 0.007$ & $1.73^{\mathrm{a}, 1} \pm 0.003$ \\
\hline \multirow[t]{4}{*}{ Wallago attu } & Copper & $1.42^{\mathrm{a}, 3} \pm 0.007$ & $0.92^{c, 6} \pm 0.005$ & $1.39^{\mathrm{b}, 4,5} \pm 0.003$ & $1.81^{\mathrm{a}, 1} \pm 0.008$ & $1.40^{c, 34} \pm 0.003$ & $1.79^{\mathrm{b}, 1,2} \pm 0.002$ \\
\hline & Chromium & $0.19^{b, 2} \pm 0.009$ & $0.18^{c, 2,3} \pm 0.007$ & $0.20^{a_{1}, 1,2} \pm 0.006$ & $0.21^{\mathrm{b}, 1} \pm 0.004$ & $0.15^{c, 4} \pm 0.002$ & $0.22^{\mathrm{a}, 1} \pm 0.005$ \\
\hline & Lead & $1.35^{\mathrm{a}, 2} \pm 0.003$ & $1.06^{c, 4} \pm 0.004$ & $1.19^{b, 3} \pm 0.007$ & $1.46^{a, 1} \pm 0.007$ & $0.87^{0,5} \pm 0.01$ & $1.44^{a, 1} \pm 0.007$ \\
\hline & Zinc & $1.43^{\mathrm{b}, 4} \pm 0.003$ & $1.20^{c, 5} \pm 0.007$ & $1.57^{a, 3} \pm 0.003$ & $1.80^{\mathrm{b}, 2} \pm 0.004$ & $1.58^{c, 3} \pm 0.007$ & $1.91^{\mathrm{a}, 1} \pm 0.004$ \\
\hline Aorichthys & Copper & $1.67^{a, 3} \pm 0.003$ & $1.17^{0,5} \pm 0.003$ & $1.62^{\mathrm{b}, 3,4} \pm 0.006$ & $1.89^{\mathrm{a}, 1} \pm 0.006$ & $1.68^{\mathrm{c}, 3} \pm 0.009$ & $1.86^{\mathrm{b}, 1,2} \pm 0.005$ \\
\hline \multirow[t]{3}{*}{ seenghala } & Chromium & $0.20^{b, 2} \pm 0.003$ & $0.17^{b, 3,4} \pm 0.004$ & $0.21^{a_{1}, 1,2} \pm 0.003$ & $0.21^{1,1,2} \pm 0.008$ & $0.18^{b, 2,3} \pm 0.003$ & $0.23^{\mathrm{a}, 1} \pm 0.007$ \\
\hline & Lead & $1.12^{\mathrm{a}, 3} \pm 0.002$ & $0.80^{c, 5} \pm 0.004$ & $1.06^{6,4} \pm 0.005$ & $1.48^{\mathrm{a}, 1} \pm 0.004$ & $1.06^{6,4} \pm 0.002$ & $1.33^{\mathrm{b}, 2} \pm 0.005$ \\
\hline & Zinc & $1.46^{b, 2} \pm 0.007$ & $1.36^{c, 3} \pm 0.006$ & $1.66^{\mathrm{a}_{1,1,2}} \pm 0.007$ & $1.49^{b, 2} \pm 0.007$ & $1.28^{\mathrm{c}, 3,4} \pm 0.002$ & $1.69^{a, 1} \pm 0.008$ \\
\hline Channa & Copper & $1.90^{a, 2} \pm 0.004$ & $1.73^{c, 3} \pm 0.006$ & $1.89^{\mathrm{b}, 2} \pm 0.005$ & $2.17^{\mathrm{a}, 1} \pm 0.003$ & $1.76^{b, 3} \pm 0.005$ & $2.14^{a, 1} \pm 0.034$ \\
\hline \multirow[t]{3}{*}{ marulius } & Chromium & $0.21^{b, 2} \pm 0.004$ & $0.18^{\mathrm{b}, 3} \pm 0.004$ & $0.24^{a, 1} \pm 0.003$ & $0.21^{\mathrm{b}, 2} \pm 0.006$ & $0.20^{c, 2,3} \pm 0.006$ & $0.25^{\mathrm{a}, 1} \pm 0.007$ \\
\hline & Lead & $1.08^{a, 3} \pm 0.003$ & $0.65^{c, 4} \pm 0.007$ & $1.02^{0,34} \pm 0.007$ & $1.47^{a, 1} \pm 0.004$ & $1.2^{6 c, 2} \pm 0.007$ & $1.42^{\mathrm{b}, 1,2} \pm 0.006$ \\
\hline & Zinc & $1.72^{\mathrm{b}, 2} \pm 0.006$ & $1.59^{c, 3} \pm 0.003$ & $1.84^{a, 1} \pm 0.003$ & $1.68^{b, 2,3} \pm 0.005$ & $1.34^{c, 4} \pm 0.002$ & $1.85^{\mathrm{a}, 1} \pm 0.005$ \\
\hline \multirow{4}{*}{$\begin{array}{l}\text { Notopterus } \\
\text { chitala }\end{array}$} & Copper & $1.87^{2,3} \pm 0.007$ & $1.56^{c, 5} \pm 0.007$ & $1.85^{\mathrm{b}, 3} \pm 0.007$ & $2.11^{\mathrm{a}, 1} \pm 0.006$ & $1.68^{b, 4} \pm 0.02$ & $2.07^{0,1,2} \pm 0.004$ \\
\hline & Chromium & $0.19^{b, 2} \pm 0.005$ & $0.14^{c, 3} \pm 0.003$ & $0.20^{\mathrm{a}, 1,2} \pm 0.003$ & $0.20^{\mathrm{b}, 1,2} \pm 0.004$ & $0.16^{c, 3} \pm 0.002$ & $0.21^{\mathrm{a}, 1} \pm 0.008$ \\
\hline & Lead & $1.32^{\mathrm{a}, 2} \pm 0.004$ & $0.83^{c, 5} \pm 0.007$ & $1.15^{b, 3} \pm 0.006$ & $1.39^{\mathrm{a}, 1} \pm 0.003$ & $0.93^{c, 4} \pm 0.005$ & $1.35^{b, 1,2} \pm 0.004$ \\
\hline & Zinc & $1.32^{\mathrm{b}, 4} \pm 0.03$ & $1.14^{c, 5} \pm 0.003$ & $1.41^{a, 3} \pm 0.003$ & $1.54^{\mathrm{b}, 2} \pm 0.002$ & $1.35^{c, 4} \pm 0.002$ & $1.72^{\mathrm{a}, 1} \pm 0.008$ \\
\hline
\end{tabular}

*Values (mean \pm standard error) in a row with different alphabetical superscripts $(a, b, c)$ differ significantly within Seasons at Site- 1 and Site- 2 and with different numerical superscripts $(1,2,3)$ differ significantly between sites $(P<0.05)$. ${ }^{*}$ Statistical analysis was carried out individually for each fish species within and between the sites

In fish, the maximum mean concentration ( $\mu \mathrm{g} \mathrm{g}^{-1}$ wet wt.) of Cu was observed in the gills of $C$. marulius (2.17 $\mathrm{ug} \mathrm{g}^{-1}$ wet wt.), followed by $N$. chitala (2.11 $\mu \mathrm{g} \mathrm{g}^{-1}$ wet wt.), A. seenghala $(1.89 \mu \mathrm{g}$ $\mathrm{g}^{-1}$ wet wt.), W. attu (1.81 $\mu \mathrm{g} \mathrm{g}^{-1}$ wet wt.) and $L$. rohita $\left(1.34 \mu \mathrm{g} \mathrm{g}{ }^{-1}\right.$ wet wt.) whereas the maximum mean concentration of $\mathrm{Pb}$ was observed in the gills of $A$. seenghala $\left(1.48 \mu \mathrm{g} \mathrm{g}^{-1}\right.$ wet wt.) followed by C. marulus (1.47 $\mathrm{ug} \mathrm{g}^{-1}$ wet wt.), W. attu (1.46 $\mu \mathrm{gg}^{-1}$ wet wt.), $N$. chitala (1.39 $\mu \mathrm{g} \mathrm{g}^{-1}$ wet wt.) and $L$. rohita $\left(0.37 \mu \mathrm{g} \mathrm{g}^{-1}\right.$ wet wt.) during pre-monsoon period whereas that of $\mathrm{Cr}$ was in the gills of C. marulius $\left(0.25 \mu \mathrm{g} \mathrm{g}^{-1}\right.$ wet wt.), followed by $A$. seenghala $(0.23$ $\mu \mathrm{g} \mathrm{g}{ }^{-1}$ wet wt.), W. attu (0.22 $\mu \mathrm{g} \mathrm{g}{ }^{-1}$ wet wt.), N. chitala $\left(0.21 \mu \mathrm{g} \mathrm{g}{ }^{-1}\right.$ wet wt.) and $L$. rohita (0.14) and of $Z n$ was observed in the gills of W. attu (1.91), followed by C. marulus (1.85), L. rohita $\left(1.73 \mu \mathrm{g} \mathrm{g}^{-1}\right.$ wet wt.), N. chitala (1.72 $\mu \mathrm{g} \mathrm{g}^{-1}$ wet wt.) and $A$. seenghala $(1.69 \mu \mathrm{g}$ $\mathrm{g}^{-1}$ wet wt.) during post-monsoon season. The mean concentrations of all the elements $(\mathrm{Cu}, \mathrm{Cr}, \mathrm{Pb}$ and $\mathrm{Zn})$ detected were below the permissible limits [ $30 \mu \mathrm{g} \mathrm{g}^{-1}, 0.5 \mu \mathrm{g} \mathrm{g}^{-1}, 0.5 \mu \mathrm{g} \mathrm{g}^{-1}$ and $40 \mu \mathrm{g} \mathrm{g}^{-1}$ respectively, recommended by FAO/WHO (1989)] in all the fish species studied except $\mathrm{Pb}$ which was found below the permissible limits in L. rohita during pre-monsoon season (Table 4). The mean concentrations of these elements in fish gills differed significantly between the sites $(p<0.05)$ during the study period. The seasonal variations in the concentration of heavy metals in fish gills was found in the following order $\mathrm{Pb}>\mathrm{Cr}>\mathrm{Cu}>\mathrm{Zn}$ according to their permissible limit.
The concentrations of all elements detected in fish tissue samples were found below the permissible limits, except for lead in fish Wallago attu at S-2 whereas in case of fish gill samples, lead was detected above the permissible limit in all the fish samples at both the sites, except in Labeo rohita. No other heavy metals were found above the permissible limits in gills. Copper at sub-lethal concentrations in fish decreases survival growth and reproductive activity the present study showed higher accumulation of $\mathrm{Cu}$ in the gills as compared to fish tissue is in conformity with the findings of Beyer et al. (2000). Anthropogenic sources of chromium emission in the surface waters are from municipal wastes, laundry chemicals, paints, leather, road runoff, agricultural runoff etc., Dixit and Tiwari (2008). Ingestion of Cr or taking it through gills and its accumulation in fish tissues and liver may occur at higher temperatures in fish (Ahmed et al., 2013). Aggarwal et al. (2000) in their study found that an increase in effluent leads to increase in the heavy metal concentration in water bodies. The seasonal changes in the concentration may be due to the fluctuation in the concentration of sewage wastes, agricultural drainage water and industrial effluents discharged into the river. It is suggested that benthic fish are likely to have higher heavy metal concentration than fish inhabiting the upper water column because they are in direct contact with the sediments and their greater uptake of heavy metal concentration from zoobenthics (Yi et al. 2011). The contamination of heavy metals may sometimes cause detrimental effects on ecology and 
diversity of aquatic fauna (Mohammadi Rouzbahani, 2017). The present study concludes that after the confluence of Buddha Nallah in river Sutlej, the concentration of heavy metals in water, sediment, fish tissues and gills altered severely and showed significantly higher values as compared to S-1 leading to higher pollution at S-2. The heavy metal accumulation and biomagnification in river systems can be detrimental not only to aquatic fauna but can also pose greater threats to humans.

\section{Acknowledgments}

The authors are highly thankful to the Dean College of Fisheries, Guru Angad Dev Veterinary and Animal Sciences University, Ludhiana, Punjab, India for providing financial support and laboratory facilities for carrying out this work. The support of the Head Department of Fisheries Resource Management and School of Veterinary Public Health and Zoonosis, College of Veterinary Sciences, Guru Angad Dev Veterinary \& Animal Sciences University, Ludhiana, Punjab, for carrying out this work is duly acknowledged.

\section{References}

Aggarwal, T. R., K. N. Singh and A.K. Gupta: Impact of sewage containing domestic waste and heavy metals on the chemistry of Varuna river water. Poll. Res., 19, 491-494 (2000).

Ahmed, M. K., G. K. Kundu., M. H. Al-Mamun., S.K. Sarkar, Akter and M. S. Khan: Chromium (VI) induced acute toxicity and genotoxicity in freshwater stinging catfish, Heteropneustes fossilis. Ecotoxico. Env., 92, 64-70 (2013).

Al-Khashman, O. A.: Determination of metal accumulation in deposited street dusts in Amman, Jordan. Env. Geochem. Hith., 29, 1-10 (2007).

Al-Yousuf, M.H., M.S. El-Shahawi and S.M. Al-Ghais: Trace metals in liver, skin and muscle of Lethrinus lentjan fish species in relation to body length and sex. Sci. Total Env., 256, 87-94 (2000).

Beyer, W. N., D. Day, M. J. Melancon and L. Sileo: Toxicity of Anacostia River, Washington DC, USA, sediment fed to mute swams (Cygnus olor). Env. Toxico. Chem., 19, 731-735 (2000).

Cevik, U., N. Damla, B. Koz and S. Kaya: Radiological characterization around the afsinelbistan coal-fired power plant in Turkey. Env. Fue., 22, 428-432 (2008).

CPCB, Central Pollution Control Board: Review of water quality objectives, requirements and zoning and classification for Indian water bodies. Government of India, Delhi, pp. 11-17 (2000).

Dalal, P., D. Chaudhry and V. Shukla: Analysis of heavy metals concentration in ambient air and in human population of Rohtak, India. J. Environ. Biol., 34, 945-949 (2013).

Dixit, S. and S. Tiwari: Impact Assessment of heavy metal pollution of Shahpura Lake, Bhopal, India. Int. J. Env. Res., 2, 37-42 (2008).

El-Sayed, A., I. El-Sayed, S. Mohamed, N. El-Sayed and Z. K. Zeinab: Assessment of heavy metals in water, sediment and fish tissues, from Sharkia province, Egypt. Egy. J. Aqua. Biol. Fishe., 15, 125-144 (2011).

FAO: Compilation of legal limits for hazardous substances in fish and fishery products. FAO Fishery Circular No. 464, Food and Agriculture Organization, pp. 5-100 (1983).

FAO/WHO: Evaluation of certain food additives and the contaminants mercury, lead and cadmium. WHO Technical Report Series No. $505(1989)$

Ikem, A., N.O. Egiebor and K. Nyavor: Trace elements in water, fish and sediment from Tuskegee lake, southeastern USA. Water, Air Soil Poll., 149, 51-75 (2003).

Miller, C.V., G.D. Foster and B.F. Majedi: Baseflow and stormflow metal fluxes from two small agricultural catchments in the coastal plain of Chesapeake Bay Basin, United States. Appl. Geo-Chem., 18, 483501 (2003).

Misra, P. C., P.C. Behera and R. K. Patel: Contamination of water due to major industries and open refuse dumping in the steel city of Orissa-A case study. J. Env. Sci. Techno., 47, 141-154 (2005).

Mohammadi Rouzbahani, M.: Bioaccumulation of heavy metals (Ni, V, $\mathrm{Cu}, \mathrm{Pb}$ ) in various tissues of Metapenaeus affinis in the Northwest of Persian Gulf. Iran. J. Aqua. Ani. Hlth, 3, 101-113 (2017).

Monteiro, V., D.G.S.M. Cavalcante, M.B.F.A. Viléla, S.H. Sofia and C.B.R. Martinez: In vivo and in vitro exposures for the evaluation of the genotoxic effects of lead on the Neotropical freshwater fish, Prochilodus lineatus. Aqua. Toxico., 104, 291-298 (2011).

Monteiro, S. M., N.M.S. Santos, M. Calejo, A. Fontainhas-Fernandes and M. Sousa: Copper toxicity in gills of the teleost fish, Oreochromis niloticus: Effects in apoptosis induction and cell proliferation. Aqua. Toxico., 94, 219-228 (2009).

Paulino, M.G., T.P. Benze, H. Sadauskas-Henrique, M.M. Sakuragui, J.B. Fernandez and M.N. Fernandes: The impact of organochlorines and metals on wild fish living in a tropical hydroelectric reservoir: Bioaccumulation and histopathological biomarkers. Sci. Total Env., 497, 293-306 (2014).

Peerzada, N. and W. Rohoza: Some heavy metals in sediments from Darwin harbour, Australia. Mari. Poll. Bulle., 20, 91-92 (1989).

Rashad, M.N.: Monitoring of environmental heavy metals in fish from Nadder Lake. Env. Int., 27, 27-33 (2001).

Reyahi-Khoram, M., F. Setayesh-Shiri and M. Cheraghi: Study of the heavy metals $(\mathrm{Cd}$ and $\mathrm{Pb})$ content in the tissues of rainbow trouts from Hamedan coldwater fish farms. Iran. J. Fish. Sci., 15, 858-869 (2016).

Sekhon, G. and B. Singh: Estimation of heavy metals in the groundwater of Patiala district of Punjab, India. Earth Reso., 1, 1-4 (2013).

Sfakianakis, D.G., E. Renieri., M. Kentouri and A.M. Tsatsakis: Effect of heavy metals on fish larvae deformities: A review. Env. Res., 137, 246-255 (2015).

Sharma, V., Y.K. Walia and A. Kumar: Assessment of physico chemical parameters for analysing water: A review. J. Biol. Chem. Chroni., 2, 25-33 (2015).

Singh, S., S. Lal, J. Harjit, S. Amlathe and H.C. Kataria: Potential of metal extractants in determination of trace metals in water sample. Adv. Stud. Biol., 3, 239-246 (2011).

USEPA.: A review of the reference doses and reference concentration processes. EPA/630/P-02/002F (2002).

WHO: World Health Organization standards for drinking water. Guidelines for Drniking Water Quality. Recommendation, France, WHO. 1, 181 (2004).

Yacoub, A. M. and N. S. Gad: Accumulation of some heavy metals and biochemical alterations in muscles of Oreochromis niloticus from the River Nile in Upper Egypt. Int. J. Env. Sci. Eng., 3, 1-10 (2012).

Yi, Y. J., Z.F. Yang and S. H. Zhang: Ecological risk assessment of heavy metals in sediment and human health risk assessment of heavy metals in fishes in the middle and lower reaches of the Yangtze River basin. Env. Poll., 159, 2575-85 (2011).

Zhou, X.D., C.L. Zhao, T.B. Qu, Y. Wang, T.J. Guo and X.G. Sun: Characteristics and evaluation on heavy metal contamination in Changchun municipal waste landfill after closure. J. Environ. Biol., 36, 857-863 (2015).

Zoumis, T., A. Schmidt, L. Grigorova and W. Calmano: Contaminants in sediments: Remobilization and demobilization. Sci. Total Env., 266, 195-202 (2001) 